\title{
Multidrug Resistance Like Protein 1 Activity in Malpighian Tubules Regulates Lipid Homeostasis in Drosophila
}

\author{
Wen Liu ${ }^{1, *,+}{ }^{\dagger}$, Hao Cao ${ }^{1,+}{ }^{+}$, Moses Kimari ${ }^{1}$, Georgios Maronitis ${ }^{1}$, Michael J. Williams ${ }^{1}$ and Helgi B Schiöth ${ }^{1,2, *}$ \\ 1 Functional Pharmacology, Department of Neuroscience, Uppsala University, 75124 Uppsala, Sweden; \\ caohao0311@163.com (H.C.); moses.kimari@neuro.uu.se (M.K.); Georgios.Karamanis@neuro.uu.se (G.M.); \\ michael.williams@neuro.uu.se (M.J.W.) \\ 2 Institute for Translational Medicine and Biotechnology, Sechenov First Moscow State Medical University, \\ 119991 Moscow, Russia \\ * Correspondence: wen.liu@neuro.uu.se (W.L.); Helgi.Schioth@neuro.uu.se (H.B.S.); \\ Fax: +46-18-471-41-36 (H.B.S.) \\ + Equal contribution.
}

check for updates

Citation: Liu, W.; Cao, H.; Kimari, M.; Maronitis, G.; Williams, M.J.; Schiöth, H.B. Multidrug Resistance Like Protein 1 Activity in

Malpighian Tubules Regulates Lipid Homeostasis in Drosophila. Membranes 2021, 11, 432. https:// doi.org/10.3390/membranes11060432

Academic Editor: Luis Octavio Regasini

Received: 30 April 2021

Accepted: 5 June 2021

Published: 8 June 2021

Publisher's Note: MDPI stays neutral with regard to jurisdictional claims in published maps and institutional affiliations.

Copyright: (C) 2021 by the authors. Licensee MDPI, Basel, Switzerland. This article is an open access article distributed under the terms and conditions of the Creative Commons Attribution (CC BY) license (https:/ / creativecommons.org/licenses/by/ $4.0 /)$.
Simple Summary: Multidrug resistance proteins (MRPs) are important for ion transport, toxin/xenobiotic secretion, and signal transduction. Although studies have been undertaken to understand their physiological function, it is not fully known how MRPs may regulate metabolism. We knocked down the expression of Drosophila multidrug-resistance like protein $1(M R P)$ in several tissues central to metabolic regulation. Reducing MRP in Malpighian tubules, the functional equivalent to the human kidney, was sufficient to disrupt metabolic homeostasis, owing to abnormal lipid accumulation, as well as changes in feeding behavior. It also increased oxidative stress resistance in adult flies, possibly due to reduced levels of reactive oxygen species.

Abstract: Multidrug resistance proteins (MRPs), members of the ATP-binding cassette transporter (ABC transporter) family, are pivotal for transporting endo- and xenobiotics, which confer resistance to anticancer agents and contribute to the clearance of oxidative products. However, their function in many biological processes is still unclear. We investigated the role of an evolutionarily conserved MRP in metabolic homeostasis by knocking down the expression of Drosophila multidrug-resistance like protein $1(M R P)$ in several tissues involved in regulating metabolism, including the gut, fat body, and Malpighian tubules. Interestingly, only suppression of MRP in the Malpighian tubules, the functional equivalent to the human kidney, was sufficient to cause abnormal lipid accumulation and disrupt feeding behavior. Furthermore, reduced Malpighian tubule MRP expression resulted in increased Hr96 (homolog of human pregnane X receptor) expression. Hr96 is known to play a role in detoxification and lipid metabolism processes. Reduced expression of MRP in the Malpighian tubules also conveyed resistance to oxidative stress, as well as reduced normal levels of reactive oxygen species in adult flies. This study reveals that an evolutionarily conserved MRP is required in Drosophila Malpighian tubules for proper metabolic homeostasis.

Keywords: lipid metabolism; ABCC1; kidney; oxidative stress; xenobiotic

\section{Introduction}

In many species, the ATP-binding cassette transporters (ABC transporters) are one of the largest transporter families. These transporters take part in cellular substrate influx and efflux. Among ABC transporters, the multidrug resistance proteins (MRPs), also known as ABCCs and GS-X pumps, belong to the $C$ subfamily and have been shown to contribute to ion transport, toxin/xenobiotic secretion, and signal transduction [1,2]. Due to the ubiquity of $M R P$ expression, numerous studies have been performed on their physiological functions $[3,4]$. 
The evolutionarily conserved MRP family member multidrug resistance protein 1 (MRP1) is widely expressed in metabolic and excretory tissues, including lung, kidney, intestines, and adipose [5,6]. In vertebrates, $M R P 1$ is well known for participating in the transport of steroid hormones, lipophilic anions, glutathione conjugates, and various drugs and xenobiotics [5-8]. However, MRP1 knockout mice are viable and fertile, indicating that MRP1 is not required for embryonic developmental processes [9]. On the other hand, abnormal MRP1 expression is remarkably relevant for various diseases and biological processes, such as hemolysis and various cancers [10-12].

The human kidney, which is the central metabolite excretion and reabsorption tissue, expresses MRP1 at high levels, and changes in MRP1 expression within the renal system are associated with chronic renal failure, inflammation, and problems with water reabsorption [13]. Moreover, human MRP1 has been reported to affect a wide range of physiological processes, including drug/xenobiotic clearance, oxidative stress, and inflammatory responses [14-16].

Recently, MRPs were shown to be involved in metabolic disruptions and syndromes in adult mice, including nonalcoholic fatty liver disease and obesity $[17,18]$. Furthermore, it was reported that MRP1 protein levels are significantly increased in the glomeruli of diabetic rats [19]. Therefore, it is reasonable to speculate that MRP proteins are associated with not only substrate transport but also other organic processes. Moreover, in a previous study, we reported that feeding Drosophila the xenobiotic dibutyl phthalate throughout larval development induces abnormal lipid accumulation, as well as causing a reduction in $M R P$ transcript levels [20]. As a crucial regulator of xenobiotic transport, $M R P$ may also contribute to disruptions in metabolic homeostasis.

Therefore, in order to better understand the function of MRP1 in the regulation of metabolic homeostasis, we employed the genetically tractable organism Drosophila melanogaster (the common fruit fly). Drosophila multidrug-resistance like protein $1(M R P)$ is highly homologous and functionally similar to human MRPs 1, 2, and 3. In Drosophila, the Malpighian tubules are the main excretory organ and are the physiological equivalent of the vertebrate kidney. Flies have four Malpighian tubules that connect at the conjunction of the midgut and hindgut, and the interaction between the intestine and the Malpighian tubules is necessary for metabolite elimination and homeostasis [21]. Similar to vertebrate kidneys, Drosophila MRP is highly expressed in the Malpighian tubules [22]. In this study, we investigated the tissue-specific effects of MRP on Drosophila metabolism.

\section{Material and Methods}

\subsection{Fly Strains and Maintenance}

The $\mathrm{w}^{*} ; \mathrm{P}\{\mathrm{w}[+\mathrm{mC}]=$ Uro-GAL4.T $\}$, (Uro-GAL4); $\mathrm{w}^{*} ; \mathrm{P}\{\mathrm{w}[+\mathrm{mW} \cdot \mathrm{hs}]=\mathrm{GawB}\} 48 \mathrm{Y},(48 \mathrm{Y}-$ GAL4); $\mathrm{w}^{*} ; \mathrm{P}\{\mathrm{w}[+\mathrm{mW} \cdot \mathrm{hs}]=\mathrm{GawB}\} \mathrm{c} 601[\mathrm{c601}],(\mathrm{c} 601-\mathrm{GAL4}) ; \mathrm{w}^{*} ; \mathrm{P}\{\mathrm{w}[+\mathrm{mC}]=\mathrm{ppl-GAL4.P}\} 2$, (ppl-GAL4), and $\mathrm{y}^{1} \mathrm{sc}^{*} \mathrm{v}^{1} ; \mathrm{P}\{\mathrm{TRiP} . H M S 01780\} a t t P 2,(U A S-M R P$ RNAi) were provided by the Bloomington Stock Center (Bloomington, IN, USA). All fly stocks and crosses were maintained on Jazz-mix Drosophila food (Fisher Scientific, Gothenburg, Sweden) and supplemented with yeast extract (Genesee Scientific, San Diego, CA, USA). These conditions will not affect the flies' lifespan. The flies were raised at $25^{\circ} \mathrm{C}$ and $60 \%$ humidity on a 12:12 h light/dark cycle. The $\mathrm{F}_{1}$ progeny were collected immediately after eclosion and aged for 5-7 days before all assays. Due to the fact the frequent reproductive cycle of the female flies could potentially influence their behavior, we used only males in our experiments.

\subsection{Knockdown of $M R P$}

MRP was knocked down in a transgenic cross and checked by performing quantitative RT-PCR (qPCR). Expression of the RNAi construct for MRP was driven by the GAL4-UAS system using the neuronal-specific elav-GAL4 driver. Thus, RNAi to MRP was expressed in all neurons. Whole male flies aged 5-7 days posteclosion were collected for analysis. The experiment was repeated five times and 10 male flies were used for each replicate. 


\subsection{Starvation Assay}

The Drosophila Activity Monitoring System (DAMS) from TriKinetics (Waltham, MA, USA) was employed to analyze the starvation resistance. Flies were collected after eclosion and aged in a vial with $8 \mathrm{~mL}$ of food. After 5-7 days of aging, each individual fly was placed in a $5 \mathrm{~mm}$ diameter tube and then monitored using the DAMS. The tubes were filled with $1 \%$ agarose $(\sim 2-3 \mathrm{~cm}$ height), which provided water and humidity but not an energy source for the flies during the assay. Then a black cap was placed on the agarose-containing end. Flies were transferred to the open side, and after they were placed in the tubes, the opening was blocked with a cotton plug. Each genotype was monitored by one DAMS, and each DAMS held 32 flies. As described, the alive/dead status of the flies was measured by the beam-crossing numbers [23]. The starvation resistance was calculated as the average survival time under starvation.

\subsection{Carbohydrate Assay}

Concentrations of body trehalose and glycogen were assessed using the Liquick CorGlucose diagnostic kit (Cormay, Marynin, Poland). At least 6 biological replicates from both control groups and the experimental group were prepared. For each replicate, $10 \mathrm{mg}$ of male flies were decapitated and homogenized in $100 \mu \mathrm{L}$ PBS buffer (pH 7.4). The samples were deproteinized at $70{ }^{\circ} \mathrm{C}$ for $5 \mathrm{~min}$, followed by incubation on ice for $5 \mathrm{~min}$. The homogenized pellets were then centrifuged at $12,900 \times \mathrm{g}$ at $4{ }^{\circ} \mathrm{C}$ for $15 \mathrm{~min}$. The supernatant was collected, and $10 \mu \mathrm{L}$ of body supernatant was mixed with either $10 \mu \mathrm{L}$ of $2 \mu \mathrm{L} / \mathrm{mL}$ porcine kidney trehalase (Sigma-Aldrich, Stockholm, Sweden) to digest trehalose or $10 \mu \mathrm{L}$ of $1 \mathrm{mg} / \mathrm{mL}$ amyloglucosidase from Aspergillus niger (Sigma-Aldrich, Stockholm, Sweden) to digest glycogen to glucose. The trehalose samples were incubated at $37^{\circ} \mathrm{C}$, and the glycogen samples were incubated at $25^{\circ} \mathrm{C}$, overnight. After incubation, $10 \mu \mathrm{L}$ of sample was diluted in $90 \mu \mathrm{L}$ of deionized water and mixed with $650 \mu \mathrm{L}$ of glucose reaction mixture (Cormay, Marynin, Poland) in a 96-well plate. Absorbance was measured with a Multiscan GO spectrophotometer (Thermo Scientific, Stockholm, Sweden) at $500 \mathrm{~nm}$. For producing the standard curve, a serial dilution was made from a $10 \mu \mathrm{g} / \mu \mathrm{L}$ standard glucose solution. The concentration was calculated according to the produced standard curve.

\subsection{Triacylglyceride (TAG) Assay}

Five flies were collected for each biological sample, and 6-8 replicates were prepared for all control and experimental groups. The flies were homogenized in $100 \mu \mathrm{L}$ of cold PBST $(\mathrm{pH}=7.2,0.05 \%$ of Tween 20$)$. After $10 \mathrm{~min}$ incubation at $70{ }^{\circ} \mathrm{C}, 20 \mu \mathrm{L}$ of the homogenized sample was separately added to either $20 \mu \mathrm{L}$ of PBST, for measuring the free glycerol, or $20 \mu \mathrm{L}$ of triglyceride reagent (Sigma-Aldrich, Stockholm, Sweden), for measuring the total glycerol content. All samples were then incubated at $37^{\circ} \mathrm{C}$ for $60 \mathrm{~min}$ and then centrifuged for $3 \mathrm{~min}$ at 14,000 rpm. After incubation, $30 \mu \mathrm{L}$ of each individual sample was transferred to a 96-well plate and mixed with $100 \mu \mathrm{L}$ of free glycerol reagent (Sigma-Aldrich, Stockholm, Sweden). The plate was incubated for $5 \mathrm{~min}$ at $37^{\circ} \mathrm{C}$. The absorbance of each sample was measured with a Multiscan GO spectrophotometer (Thermo Scientific, Stockholm, Sweden) at $540 \mathrm{~nm}$ and calculated according to the standard curve. The standard curve was obtained from a serial dilution of glycerol standards (Sigma-Aldrich, Stockholm, Sweden) and was produced along with the samples. The TAG concentration was determined as $T A G$ concentration $=$ total glycerol concentration - free glycerol concentration .

\subsection{Fly Proboscis and Activity Detector (FlyPAD)}

We performed FlyPAD experiments using starved flies because the food pellet can only hold a small amount of food ( $150 \mathrm{mM}$ sucrose, only 2-3 microliters), which will dry out within $2 \mathrm{~h}$, resulting in feeding times of usually no more than $2 \mathrm{~h}$. Fed flies usually eat slowly; therefore, we chose to use flies starved for $18 \mathrm{~h}$ instead. The assay was adapted from Itskov et al. [24]. In brief, male flies from both control and experimental crosses were collected after eclosion and aged for 5 days, under a $12: 12 \mathrm{~h} \mathrm{light} /$ dark cycle, at $25^{\circ} \mathrm{C}$. The 
assay was conducted between 8 a.m. and 11 a.m. Before the experiment was performed, flies were transferred into the flyPAD arena (flies were not anesthetized before transfer). Each arena included two detective channels, one with a $150 \mathrm{mM}$ sucrose (dissolved in $1 \%$ agarose) pellet and one that was blank. All the interactions between the flies and the food pellet were monitored by Bonsai software. Thirty-two replicates were used for each group. Data were analyzed by using the MATLAB-based graphic user interface (GUI) program.

\subsection{ROS Detection}

Reactive oxygen species were monitored using the Amplex Red Hydrogen Peroxide/Peroxidase Assay Kit, according to the instruction provided by the manufacturer (Molecular Probes, Eugene, OR, USA). Six fly bodies were homogenized in phosphate buffer ( $\mathrm{pH} 7.4$ ) and used as one replicate. The reaction mixture contained $50 \mu \mathrm{M}$ Amplex Red reagent and $0.1 \mathrm{U} / \mathrm{mL}$ horseradish peroxidase (HRP). Fluorescence was recorded at $530 \mathrm{~nm}$ excitation/587 $\mathrm{nm}$ emission. The results were normalized using Uro-GAL4 > $w^{1118}$ control flies.

\subsection{Paraquat Resistance}

Flies were collected and raised in a vial containing $8 \mathrm{~mL}$ of normal fly food at $25{ }^{\circ} \mathrm{C}$ for 5 posteclosion days and then transferred to a vial containing 1\% agarose for $6 \mathrm{~h}$ starvation in order to eliminate all the remaining food. Paraquat (Sigma-Aldrich, Stockholm, Sweden) was dissolved in $10 \%(w / v)$ sucrose solution at a final concentration of $20 \mathrm{mM}$. A round filter paper soaked with paraquat solution was placed in a new agarose vial, and 13-15 flies for each replicate were transferred to this vial after starvation; 6-8 biological replicates were prepared. To assess the resistance to paraquat, the survival rate was recorded 3 times per day until all flies died, and both the filter papers and the agarose vials were replaced daily.

\subsection{Quantitative Real-Time PCR}

Six replicates containing 10 decapitated fly bodies for each group were prepared. The RNA was extracted by using the TRIzol reagent (Invitrogen, Stockholm, Sweden). cDNA reverse transcription was performed by using a High-Capacity RNA-to-cDNA kit (Applied Biosystems, Foster City, CA, USA). Approximate $10 \mathrm{ng}$ of cDNA sample was used as a template for each replicate and amplified with Taq DNA polymerase (Biotools) in Bio-IQ5 cycler (Bio-Rad, Solna, Sweden). Rp49 was used as the reference gene. The primer sequences used were as follows:

- Rp49-F: 5'-CACACCAAATCTTACAAAATGTGTGA-3';

- Rp49-R: 5'-AATCCGGCCTTGCACATG-3';

- Hr96-F: 5'-GATATGTTCCTCCAGGCCCTA-3';

- Hr96-R: 5'-TGTGCGTGGCAAAGAAGACT-3';

- Cnc-F: 5'-CTGCATCGTCATGTCTTCCAGT-3';

- Cnc-R: 5'-AGCAAGTAGACGGAGCCAT-3';

- Keap1-F: 5'-AGGCCAATGTGTTTATTGAGCG-3';

- Keap1-R: 5'-GCAATCAACTGATATGCCGAAAG-3';

- ss-F: 5'-GATATGTTCCTCCAGGCCCTA-3';

- $\quad$ ss-R: $5^{\prime}$-TGTGCGTGGCAAAGAAGACT-3';

- MRP-R: 5'-GAATCTGGGTCTGCTGGTAATC;

- MRP-R: 5'-AAACATCCAGGTCGTAGAGCG.

\section{Data Analysis}

Data analysis and plotting were performed using Prism GraphPad 5. Data are presented as mean \pm SEM. Except for the paraquat survival test, all the significant differences were tested following one-way ANOVA with Tukey's post hoc test. For evaluating the paraquat resistance, the comparisons were analyzed by Kaplan-Meier log-rank test. The significances are represented as ${ }^{*} p<0.05,{ }^{* *} p<0.01$, and ${ }^{* * *} p<0.001$ and are also specified in the corresponding figure legends. 


\section{Results}

\subsection{Successful Knockdown of MRP}

In order to determine if the RNAi line was functional, we crossed the UAS-MRP RNAi line with elav-GAL4 flies, to express the RNAi line on all neurons, and performed quantitative RT-PCR (qPCR) on the adult $\mathrm{F}_{1}$ progeny. Supplementary Figure $\mathrm{S} 1$ shows that MRP was successfully knocked down in the transgenic cross.

\subsection{Loss of MRP in Malpighian Tubules Increases Starvation Resistance}

Drosophila MRP is highly expressed in the gut and Malpighian tubules and at lower levels in the fat body $[22,25]$. To study the function of $M R P$ in different organs, we crossed UAS-MRP RNAi flies with four tissue-specific GAL4 drivers, namely a mid-gut driver (48Y-GAL4), a hind-gut driver (c601-GAL4), a fat body driver (ppl-GAL4), and a Malpighian tubule driver (Uro-GAL4), and then performed a starvation resistance assay using 5-7day-old male flies from the $\mathrm{F}_{1}$ generation. It has been reported that $M R P$ is expressed in all of these tissues [25]. The starvation resistance assay can potentially uncover any effects on energy utilization, lipid storage, and feeding behavior. Notably, we observed that none of the 48Y-GAL4, c601-GAL4, and ppl-GAL4 crosses exhibited a starvation resistance phenotype when compared to control flies (Figure 1A-C). However, knocking down MRP in the Malpighian tubules (Uro-GAL4 > UAS-MRP RNAi) significantly increased resistance to starvation when compared to control flies $(61.57 \pm 1.16 \mathrm{~h}$ for Uro-GAL4 $>$ UAS-MRP RNAi vs. $56.66 \pm 0.85 \mathrm{~h}$ for Uro-GAL4 $>w^{1118}(p<0.05)$ and vs. $54.90 \pm 1.47 \mathrm{~h}$ for $w^{1118}>$ UAS-MRP RNAi $(p<0.001)$; Figure 1D).
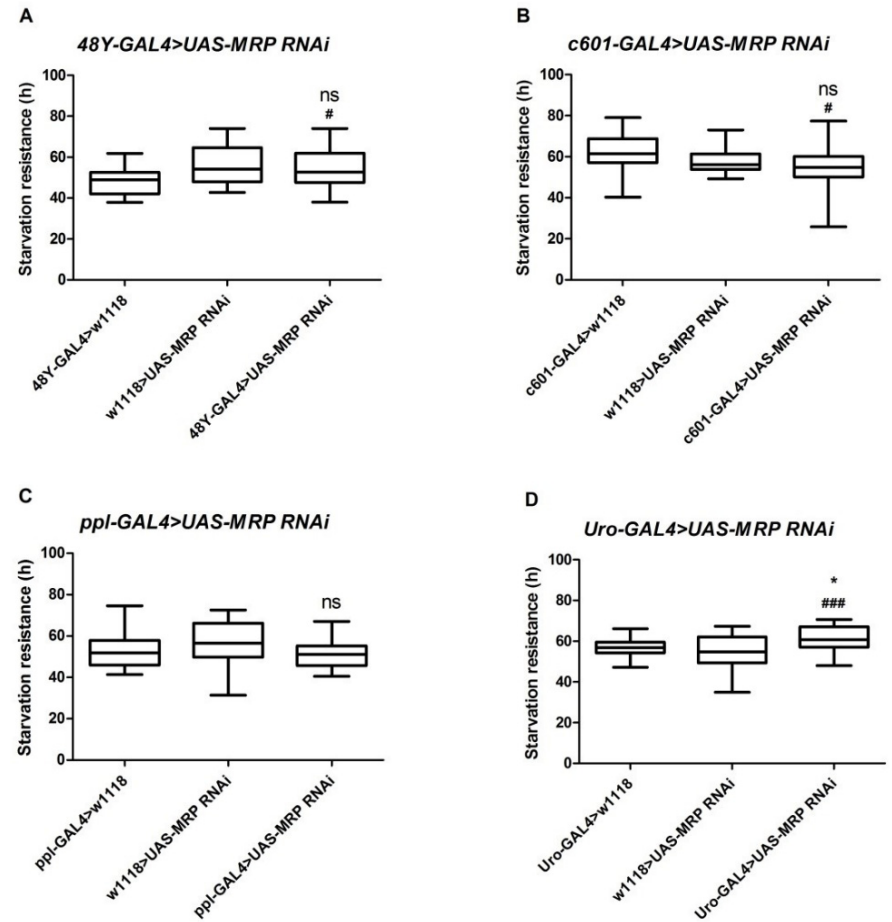

Figure 1. Crossing MRP RNAi with four different tissue-specific drivers. Graphs are presented in box plots, showing starvation resistance of both experimental and control groups. All Gal4 drivers were crossed with a UAS-MRP RNAi line: (A) 48Y-GAL4 (the mid-gut driver), (B) c601-GAL4 (the hind-gut driver), (C) ppl-GAL4 (the fat body driver), and (D) Uro-GAL4 (the Malpighian tubule driver). Error bar represents the max and min values, and 32 male flies were used for each individual crossed strain; ns, not significant compared to either $w^{1118}>$ UAS-MRP RNAi or both of the controls; ${ }^{\#} p<0.01$, compared to GAL4-driver $>w^{1118}$; \#\# $p<0.001$, compared to Uro-GAL4 $>w^{1118} ;{ }^{*} p<0.01$, compared to $w^{1118}>$ UAS-MRP RNAi flies; one-way ANOVA with Tukey's post hoc test was performed. 


\subsection{Loss of MRP in Malpighian Tubules Influences Triacylglyceride Levels}

The starvation resistance defect, found in Uro-GAL4 > UAS-MRP RNAi flies, established a possibility for metabolic disruption. Therefore, we measured the carbohydrate and triacylglyceride (TAG) levels of adult flies. The levels of circulating glucose (Figure 2A), circulating trehalose (Figure 2B), stored trehalose (Figure 2C), or glycogen (Figure 2D), were not significantly affected by the reduction in MRP expression in Malpighian tubules. However, the TAG content of the experimental group $(2.44 \pm 0.29 \mathrm{mg} / \mathrm{mL}, p<0.05$, Figure 2E) was significantly elevated compared to control groups $(1.23 \pm 0.08 \mathrm{mg} / \mathrm{mL}$ for Uro-GAL4 $>w^{1118}$ and $0.57 \pm 0.08 \mathrm{mg} / \mathrm{mL}$ for $w^{1118}>$ UAS-MRP RNAi).
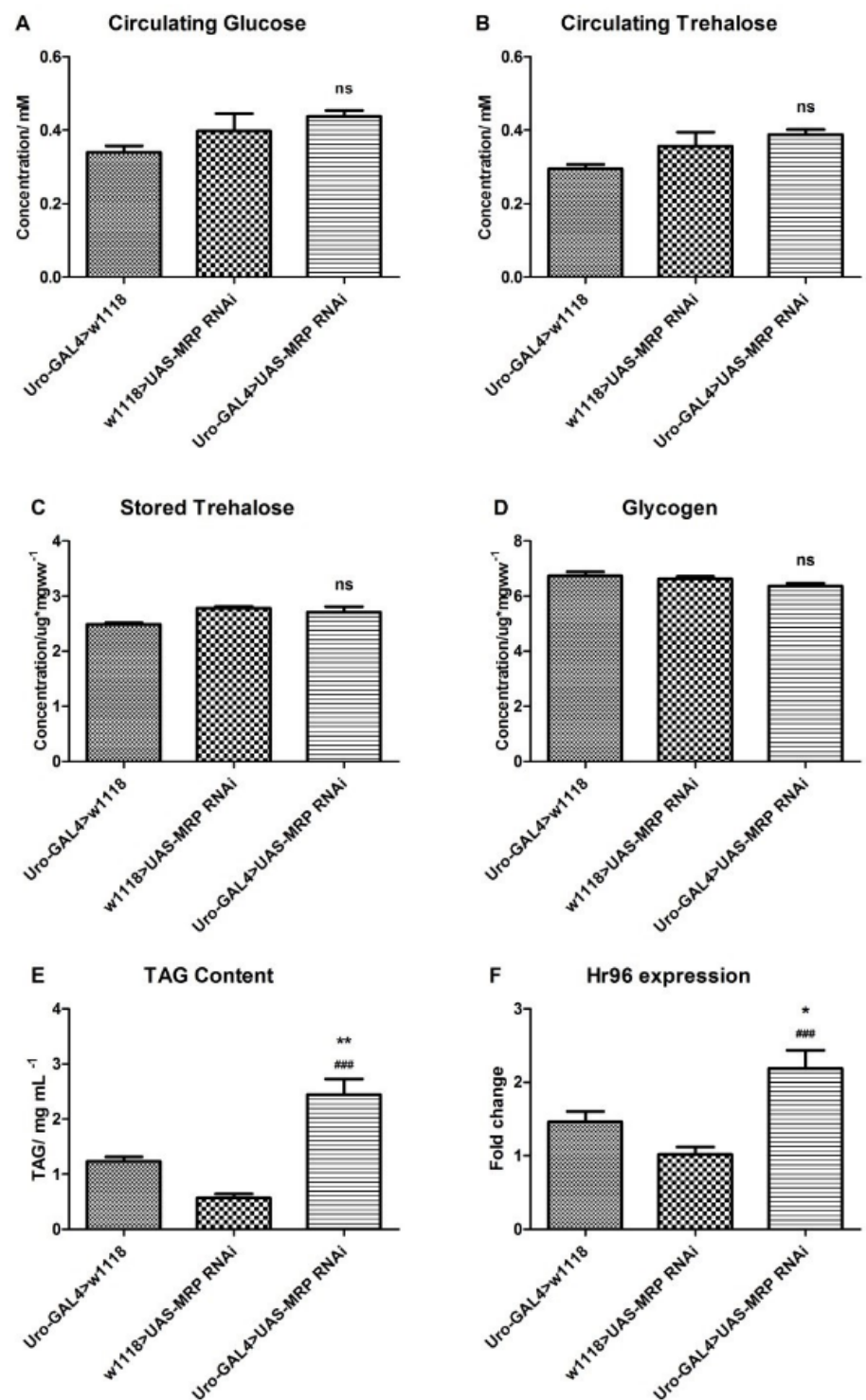

Figure 2. $M R P$ knockdown in the Malpighian tubules affects lipid content. Flies for the carbohydrate assay were either fed ad libitum or starved for $24 \mathrm{~h}$ before the assay. Uro-GAL4 > UAS-MRP RNAi flies exhibited no effects on their (A) circulating glucose, (B) circulating trehalose, $(\mathbf{C})$ stored trehalose, and (D) glycogen. However, MRP knockdown in the Malpighian tubules induced a significant increase in (E) triacylglyceride (TAG) content. The mRNA expression of the xenobiotic sensing receptor, Hr96, was notably increased (F). All graphs are presented as mean \pm SEM; 10-15 male flies for carbohydrate assay and 6 male flies for TAG assay were used per sample, and 4-6 biological samples were prepared for each assay; ns, not significant compared to either of the control groups; ${ }^{* *} p<0.01$ and ${ }^{*} p<0.05$, compared to Uro-GAL4 > w $w^{1118}$; \#\#\# $p<0.001$, compared to $w^{1118}>$ UAS-MRP RNAi flies; one-way ANOVA with Tukey's post hoc test was performed. 
Sieber et al. reported that $\mathrm{Hr} 96$, the Drosophila homolog of human nuclear receptor subfamily 1, group I, member 2 (NR1I2, also known as pregnane $X$ receptor $(P X R)$ ), was involved in lipid, but not carbohydrate, homeostasis in fruit flies [26]. Furthermore, as a phase II enzyme in xenobiotic transportation, NR1I2 has been shown to interact with the ABC transporter family $[27,28]$. Moreover, we have shown that similar to $M R P$, exposure of larva to the xenobiotic dibutyl phthalate significantly reduced $\mathrm{Hr} 96$ transcript levels [20]. Therefore, using qPCR, we examined Hr96 expression in whole adult flies where MRP was knocked down in the Malpighian tubules. Compared to controls, $\mathrm{Hr} 96$ expression increased significantly when $M R P$ was knocked down in the Malpighian tubules (approximately 1.1-fold increase compared to $w^{1118}>U A S-M R P$ RNAi group $(p<0.001)$ and 0.5-fold increase compared to Uro-GAL4 $>w^{1118}$ group $(p<0.05)$ ) (Figure $\left.2 \mathrm{~F}\right)$.

\subsection{Loss of MRP in the Malpighian Tubules Influences Feeding Behavior}

Since we detected deficiencies in starvation resistance and lipid metabolism, we determined if MRP knockdown in Malpighian tubules might also influence feeding behavior. To do this, we employed the Fly Proboscis and Activity Detector (flyPAD). The flyPAD enables real-time recording of food intake and feeding behavior with a high temporal resolution [24]. As a consequence of $M R P$ knockdown in Malpighian tubules, the Uro-GAL4 > UAS-MRP RNAi flies exhibited an obvious reduction in total food intake, which was represented by the total number of sips (bites) (226.9 \pm 12.5 sips for UroGAL4 $>w^{1118}$ and $224.2 \pm 28.4$ sips for $w^{1118}>U A S-M R P$ RNAi vs. $112.5 \pm 12.4$ sips for Uro-GAL4 > UAS-MRP RNAi, $p<0.005$ ) (Figure 3A). Additionally, their activity bouts, which reflect all interactions of the flies with the food pellet, and feeding bursts, which denote the number of meals flies have during the monitoring, were decreased. This indicated that experimental flies had significantly fewer meals and interactions with the food (for the comparison of all the groups, $p<0.01$ ) (Figure 3B,C). Of note, although the number of sips per feeding burst (Figure 3D) and the duration of each activity bout (Figure 3E) were unaffected, the meal duration (feeding burst) increased in the experimental flies (for the comparison of all the groups, $p>0.05$ ) (Figure 3F).

\subsection{MRP Knockdown in Malpighian Tubules Confers Oxidative Resistance to Drosophila melanogaster}

MRP transporters are tightly related to oxidative responses [8,29], and a reduction in oxidative resistance may contribute to imbalanced metabolic homeostasis. Therefore, we performed an oxidative resistance assay by exposing flies to paraquat-containing food. Paraquat is a commonly used herbicide that can induce oxidative stress when administrated to flies [30]. The surviving flies were counted, and the survival rate was calculated daily; these data reflect the degree to which the flies were resistant to oxidative stress. To our surprise, the oxidative resistance of MRP knockdowns was not diminished, as we had assumed. In contrast, the Uro-GAL4 > UAS-MRP RNAi flies showed increased resistance to oxidative stress. Unlike a report that showed the detectable susceptibility to oxidants of global Drosophila MRP4 knockdowns [31], the Malpighian tubule specific MRP knockdown flies were more resistant to oxidative stress. The experimental flies survived significantly longer than both control groups (the median survival was $110 \mathrm{~h}$ for Uro-GAL4 $>w^{1118}$ and $68 \mathrm{~h}$ for $w^{1118}>U A S-M R P$ RNAi, while the value was $136 \mathrm{~h}$ for Uro-GAL4 > UAS-MRP RNAi, and the $p$-values were less than 0.01 and 0.001 , respectively) (Figure 4A). In order to confirm this possibility, we conducted a reactive oxygen species (ROS) detection assay using freshly made whole-body homogenate. Consistent with the paraquat assay, the Uro-GAL4 > UAS-MRP RNAi flies exhibited less ROS production than controls (Figure 4B). 
A

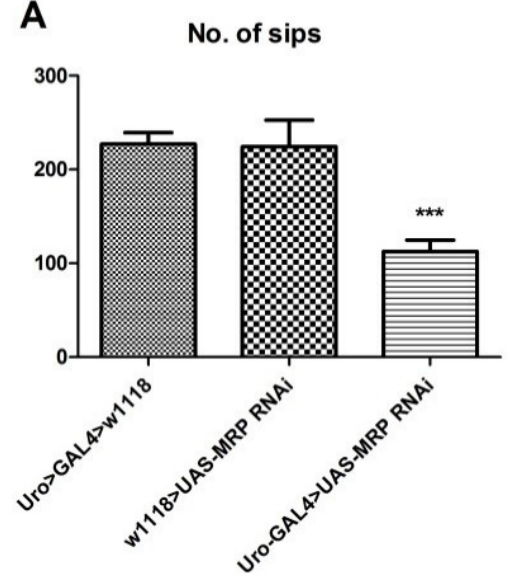

D

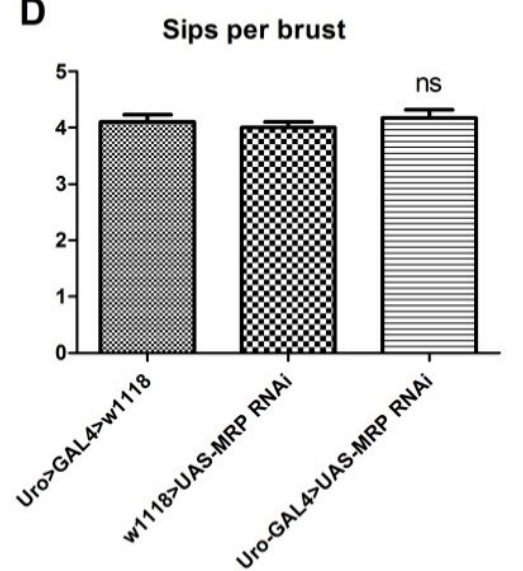

B

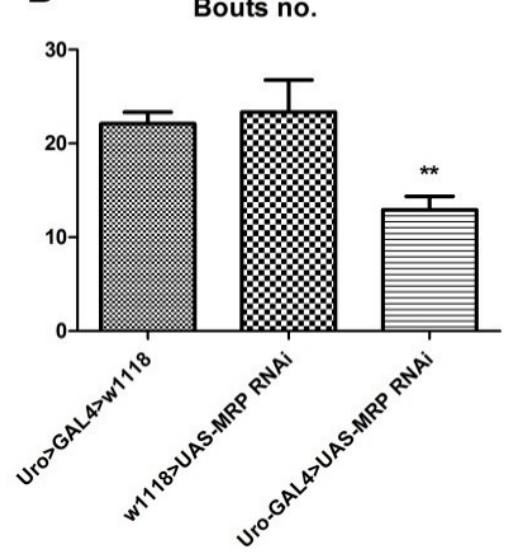

E

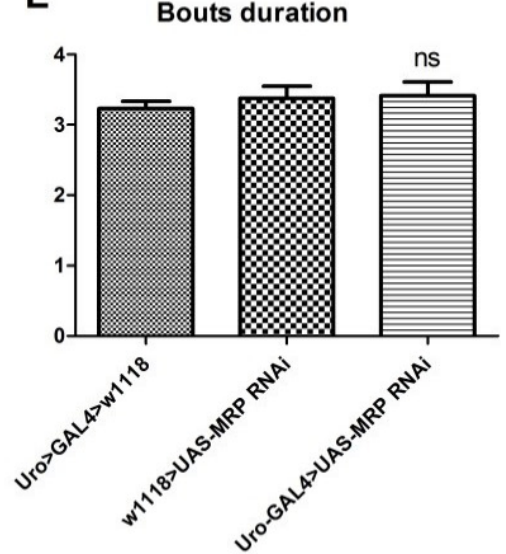

C

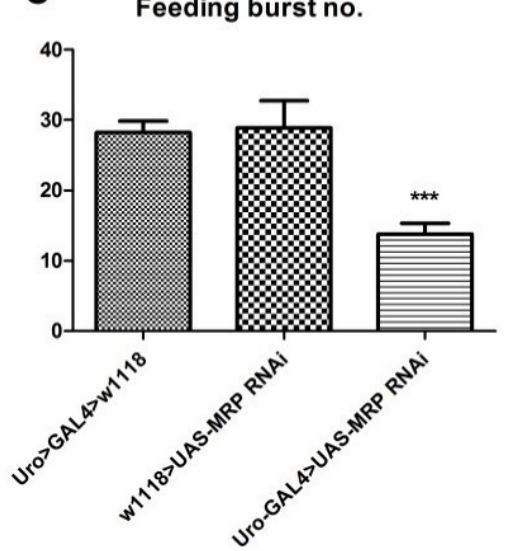

$\mathbf{F}$

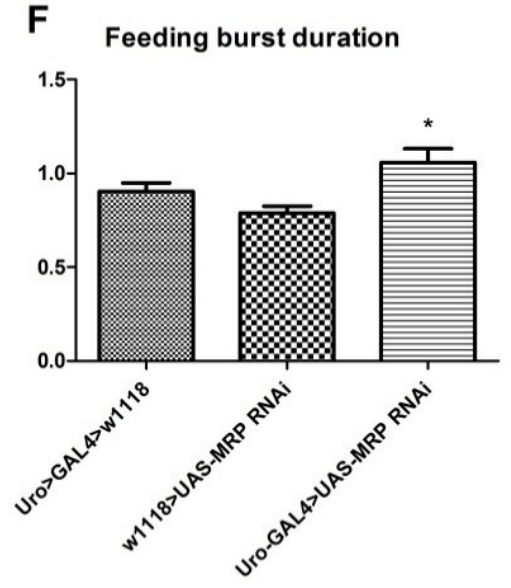

Figure 3. MRP knockdown in the Malpighian tubules affects feeding behavior. Male flies 5-7 days of age were used, and the flyPAD was employed to assess the feeding behavior. (A) The number of sips, which represents the total food intake; (B) the number of feeding bouts, which represents any interaction with food drop; and (C) the number of feeding bursts, which represents the number of meals, were all significantly decreased in the experimental group. However, both (D) the sips per burst and (E) bout duration were not affected. The feeding burst (F) duration was slightly increased in the experimental group. All graphs are presented as mean $\pm \mathrm{SEM}, n=32$ males per group; ns, not significant; ${ }^{*} p<0.05$, ${ }^{* *} p<0.01,{ }^{* * *} p<0.001$; one-way ANOVA with Tukey's post hoc test was performed; the experimental group was compared to each of the control groups.

Next, we used qPCR to examine the expression levels of genes associated with oxidative stress, including ss (spineless, the Drosophila homolog of aryl hydrocarbon receptor $(A h R))$ [32], cnc (cap-n-collar, the Drosophila homolog of NF-E2-related factor 2 (NRF2)), and Keap1 (Kelch-like ECH associated protein 1) [33,34]. The expression of ss, but not cnc or Keap1, was significantly increased in the MRP Malpighian tubule knockdowns (1.56 \pm 0.16 -fold change for Uro-GAL4 > UAS-MRP RNAi, compared to $1.02 \pm 0.07$-fold change for Uro-GAL4 $>w^{1118}$ and $0.80 \pm 0.05$-fold change for $w^{1118}>$ UAS-MRP RNAi) (Figure 4C). 

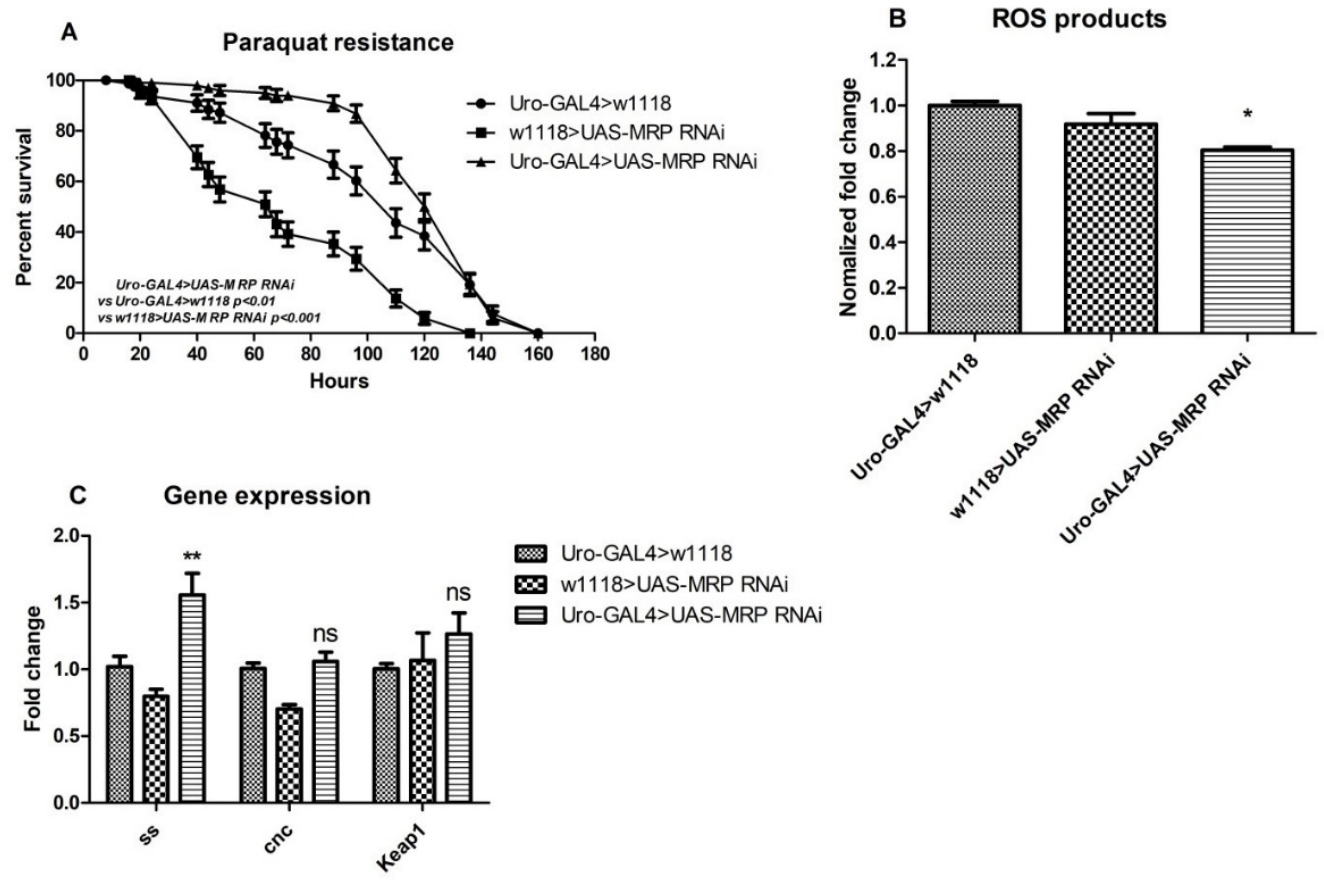

Figure 4. Malpighian tubule specific MRP knockdown conferred oxidative stress resistance. Flies 5-7 days of age were used. (A) Thirteen to fifteen flies were placed in a vial with a filter paper soaked with paraquat-sucrose solution. The death of flies was registered 3 times per day. The Uro-GAL4 > UAS-MRP RNAi flies exhibited more resistance to paraquat than both of the control groups (6-8 replicates were used for each group of flies; data are represented as percentage survival \pm SEM; $p$ values were shown on the plot; Kaplan-Meier log-rank test was performed). (B) ROS production of the Uro-GAL4 > UAS-MRP RNAi flies was significantly less than that of both of the controls, which indicates diminished oxidative stress level. Six flies were used for each replicate and 6 replicates were prepared for each group. The data were normalized to the Uro-GAL4 $>w^{1118}$ group. (C) The expression of ss, cnc, and Keap1 were tested using qPCR. Although no difference was observed in $c n c$ and Keap1 expression, ss expression levels increased significantly in the experimental flies. The graph represents as mean $\pm \mathrm{SEM}{ }^{*} p<0.05,{ }^{* *} p<0.01$; one-way ANOVA with Tukey's post hoc test was performed.

\section{Discussion}

We determined that a specific reduction in MRP expression within the Drosophila Malpighian tubules is sufficient to increase triacylglycerol (TAG) levels in adult flies. Reduced MRP expression in the Malpighian tubules also disrupts normal feeding behavior and increases resistance to oxidative stress. This study highlights that $\mathrm{ABC}$ transporters, which are generally reported to be more involved in drug, xenobiotic, and ion transport, may also be important for regulating biochemical metabolites, such as lipids [35].

We also provide some evidence that $M R P$ is an indispensable molecule in biochemical metabolism in Drosophila. To study the tissue-specific functions of $M R P$, we employed the efficient GAL4-UAS system [36] in order to knock down MRP in the mid-gut, hind-gut, fat body, or Malpighian tubules. Interestingly, we found that when MRP was knocked down in the Malpighian tubules, which are functionally similar to the mammalian kidney, flies were significantly more resistant to starvation. This indicates the possible importance of MRP in metabolic homeostasis. In Drosophila a relationship between the Malpighian tubules and metabolic signaling has been reported. Söderberg et al. reported that insulin signaling within the Malpighian tubules was important for responses to metabolic stress [37]. Moreover, in the same article, knocking down tachykinin-like receptor at 99D (TkR99D) in Malpighian tubules led to increased resistance to both desiccation and starvation. In another study, MRP was shown to participate in tachykinin-evoked ATP release [38]. Taken together, it is reasonable to speculate that in the Malpighian tubules, MRP might contribute to resisting metabolic stress by regulating energy balance and metabolic homeostasis.

Encouraged by our starvation findings, we measured the carbohydrate and lipid contents of flies in which MRP expression was reduced in the Malpighian tubules. As a 
consequence of $M R P$ reduction, TAG levels were significantly higher than in the control flies. In fact, MRP-mediated lipid transport has been reported in mammals. Raggers et al. reported that MRP1 transfected pig kidney had increased short-chain sphingolipid outward transport [39]. The evidence of lipid transport mediated by MRP was also confirmed by Kamp and Haest, who reported that the presence of MRP could be implicated in phospholipid outward transport [40]. Furthermore, MRP family members have been shown to transport cholesterol-derived corticosteroids [6]. Therefore, it is possible that Drosophila MRP mediates lipid homeostasis.

The human PXR/CAR homolog in insects, $\mathrm{Hr}$ 96, has been reported [41] to control lipid homeostasis in Drosophila [26,42]. As a xenobiotic-sensing nuclear receptor, NR1I2/PXR activation was able to alter energy utilization and lipid allocation, which eventually leads to metabolic disorders $[43,44]$. Recent studies have demonstrated that NR1I2 / PXR regulates the expression of key proteins involved in endobiotic responses, such as the metabolic homeostasis of lipids, glucose, and bile acid (OLADIMEJI et al., 2018). Furthermore, a connection between NR1I2/PXR and MRP was found in human hepatocytes [45], and $M R P$ was shown to be regulated by PXR/CAR (Wei et al., 2000; Maglich et al., 2002). Here, we provide another clue regarding an interaction between $H r 96$ and $M R P$, where the expression of $\mathrm{Hr} 96$ was affected by the reduction in MRP specifically in the Malpighian tubules. Subsequently, we found that feeding behavior and food intake are influenced by Malpighian tubule $M R P$, which could be a result of MRP-induced lipid accumulation disrupting energy balance and inhibiting food intake and feeding patterns.

We also observed that oxidative responses were strengthened by $M R P$ deficiency in the Malpighian tubules (see Figure 4). Considering that glutathione and its conjugates, some of the main substrates of MRPs, are crucial in oxidative responses, we chose to evaluate the role of Malpighian tubule expressed MRP in oxidative stress. Two investigations carried out by Takahashi et al. indicated that MRP is important in protecting endothelial cells against oxidative stress [46,47]. Furthermore, another Drosophila study validated that an MRP4 mutation could suppress general oxidative resistance and contribute to lifespan elongation [31]. In contrast, a recent study illustrated that $M R P 1^{-/}$mutant mice had reduced ROS production in a streptozotocin-induced diabetic model and suggested MRP1 as a novel target for diabetes treatment [10]. Different from the global mutation, our findings show that interfering with MRP expression in the Malpighian tubules is sufficient to confer oxidative stress resistance. We then tested genes known to be highly regulated by the oxidative response. Although the Keap1/Nrf2 signal mediates the cellular oxidative response and xenobiotic elimination [48,49], no change in the expression of these genes was detected in MRP Malpighian tubule knockdown flies. However, when MRP was knocked down specifically in Malpighian tubules, the expression of the human $A h R$ homolog spineless (ss) was significantly upregulated. AHR is a transcription factor known to regulate the cytochrome P450 genes and participate in phase I xenobiotic clearance in mammals [50,51]. There are reports showing that mice treated with AHR inducers exhibited increased expression of MRPs in hepatic cells [52,53]. Thus, a defect in MRP function, a part of the phase III xenobiotic clearance system, might cause the compensatory overexpression of $s s$ in flies, and the activation of $s s(A h R)$ may be necessary to protect cells under oxidative stress. Therefore, knocking down MRP in Malpighian tubules could lead to compensatory ss expression, which then contributes to oxidative resistance in flies.

\section{Conclusions}

Our present study reveals a role for Malpighian tubule expressed $M R P$ in metabolic regulation in Drosophila. The tissue-specific deficiency of MRP led to overall lipid accumulation and changes in the feeding pattern of Drosophila adult males. Furthermore, inadequate $M R P$ expression results in an inhibition of oxidative stress and ROS production. Altogether, these findings provide a new insight to guide future studies of MRP in metabolic homeostasis. 
Supplementary Materials: The following are available online at https://www.mdpi.com/article/10 $.3390 /$ membranes11060432/s1, Figure S1: Successful knockdown of MRP. MRP was knocked down in a transgenic, cross. Expression of an RNAi construct for MRP was driven by the GAL4-UAS system using the neruomal specific elav-GAL4 driver. Whole male flies which aged 5-7 days post-eclosion were collected for qPCR analysis. The experiment was repeated five times and 10 male flies were used for each replicate. The data were presented as mean \pm SEM.

Author Contributions: Data curation, W.L. and H.C.; Formal analysis, H.C.; Funding acquisition, H.B.S.; Investigation, H.C.; Methodology, W.L., H.C., M.K., G.M. and M.J.W.; Project administration, W.L., M.J.W. and H.B.S.; Supervision, M.J.W.; Validation, M.J.W. and H.B.S.; Visualization, H.B.S.; Writing-original draft, W.L.; Writing—review \& editing, W.L., M.J.W. and H.B.S. All authors have read and agreed to the published version of the manuscript.

Funding: This research was funded by: Swedish Research Council [2018-03238], Formas (the Swedish government research council for sustainable development): [2018-00738], Swedish Cancer Foundation (CancerFonden): [20 0990 Pj], the Swedish Brain Research Foundation, Novo Nordisk Foundation, FAT4BRAIN project funding from the European Union's Horizon 2020 research and innovation program (grant \#857394).

Institutional Review Board Statement: Not applicable.

Informed Consent Statement: Not applicable.

Data Availability Statement: The authors confirm that the data supporting the findings of this study are available within the article.

Acknowledgments: We wish to thank all of the laboratory members for helpful discussions. H.B.S. was supported by the Swedish Research Council, the Swedish Brain Research Foundation, the Formas (the Swedish government research council for sustainable development), the Novo Nordisk Foundation, the Swedish Cancer Foundation, and the FAT4BRAIN project funding from the European Union's Horizon 2020 research and innovation program (grant \#857394). Hao Cao was supported by the China Scholarship Council.

Conflicts of Interest: The authors declare that they have no conflict of interest.

\section{References}

1. Gu, X.; Manautou, J.E. Regulation of hepatic ABCC transporters by xenobiotics and in disease states. Drug Metab. Rev. 2010, 42, 482-538. [CrossRef]

2. Keppler, D. Multidrug resistance proteins (MRPs, ABCCs): Importance for pathophysiology and drug therapy. Handb. Exp. Pharmacol. 2011, 299-323. [CrossRef]

3. Leslie, E.M.; Deeley, R.G.; Cole, S.P. Multidrug resistance proteins: Role of P-glycoprotein, MRP1, MRP2, and BCRP (ABCG2) in tissue defense. Toxicol. Appl. Pharmacol. 2005, 204, 216-237. [CrossRef] [PubMed]

4. Glavinas, H.; Krajcsi, P.; Cserepes, J.; Sarkadi, B. The role of ABC transporters in drug resistance, metabolism and toxicity. Curr. Drug Deliv. 2004, 1, 27-42. [CrossRef] [PubMed]

5. Deeley, R.G.; Cole, S.P. Substrate recognition and transport by multidrug resistance protein 1 (ABCC1). FEBS Lett. 2006, 580, 1103-1111. [CrossRef] [PubMed]

6. Nixon, M.; Mackenzie, S.D.; Taylor, A.I.; Homer, N.Z.; Livingstone, D.E.; Mouras, R.; Morgan, R.A.; Mole, D.J.; Stimson, R.H.; Reynolds, R.M.; et al. ABCC1 confers tissue-specific sensitivity to cortisol versus corticosterone: A rationale for safer glucocorticoid replacement therapy. Sci. Transl. Med. 2016, 8, 352ra109. [CrossRef]

7. Ishikawa, T.; Kuo, M.T.; Furuta, K.; Suzuki, M. A new aspect on glutathione-associated biological function of MRP/GS-X pump and its gene expression. Cytotechnology 1998, 27, 81-93. [CrossRef] [PubMed]

8. Hipfner, D.R.; Deeley, R.G.; Cole, S.P. Structural, mechanistic and clinical aspects of MRP1. Biochim. Biophys. Acta 1999, 1461, 359-376. [CrossRef]

9. Wijnholds, J.; Evers, R.; van Leusden, M.R.; Mol, C.A.; Zaman, G.J.; Mayer, U.; Beijnen, J.H.; van der Valk, M.; Krimpenfort, P.; Borst, P. Increased sensitivity to anticancer drugs and decreased inflammatory response in mice lacking the multidrug resistance-associated protein. Nat. Med. 1997, 3, 1275-1279. [CrossRef]

10. Neuser, J.; Fraccarollo, D.; Wick, M.; Bauersachs, J.; Widder, J.D. Multidrug resistance associated protein-1 (MRP1) deficiency attenuates endothelial dysfunction in diabetes. J. Diabetes Complicat. 2016, 30, 623-627. [CrossRef]

11. Vander Borght, S.; Komuta, M.; Libbrecht, L.; Katoonizadeh, A.; Aerts, R.; Dymarkowski, S.; Verslype, C.; Nevens, F.; Roskams, T. Expression of multidrug resistance-associated protein 1 in hepatocellular carcinoma is associated with a more aggressive tumour phenotype and may reflect a progenitor cell origin. Liver Int. 2008, 28, 1370-1380. [CrossRef] 
12. Nowicki, M.T.; Aleksunes, L.M.; Sawant, S.P.; Dnyanmote, A.V.; Mehendale, H.M.; Manautou, J.E. Renal and hepatic transporter expression in type 2 diabetic rats. Drug Metab. Lett. 2008, 2, 11-17. [CrossRef]

13. van de Water, F.M.; Masereeuw, R.; Russel, F.G. Function and regulation of multidrug resistance proteins (MRPs) in the renal elimination of organic anions. Drug Metab. Rev. 2005, 37, 443-471. [CrossRef]

14. Hirrlinger, J.; Konig, J.; Keppler, D.; Lindenau, J.; Schulz, J.B.; Dringen, R. The multidrug resistance protein MRP1 mediates the release of glutathione disulfide from rat astrocytes during oxidative stress. J. Neurochem. 2001, 76, 627-636. [CrossRef]

15. Cole, S.P. Multidrug resistance protein 1 (MRP1, ABCC1), a "multitasking" ATP-binding cassette (ABC) transporter. J. Biol. Chem. 2014, 289, 30880-30888. [CrossRef]

16. Zhang, Y.K.; Wang, Y.J.; Gupta, P.; Chen, Z.S. Multidrug Resistance Proteins (MRPs) and Cancer Therapy. AAPS J. 2015, 17, 802-812. [CrossRef] [PubMed]

17. Ditzel, E.J.; Li, H.; Foy, C.E.; Perrera, A.B.; Parker, P.; Renquist, B.J.; Cherrington, N.J.; Camenisch, T.D. Altered Hepatic Transport by Fetal Arsenite Exposure in Diet-Induced Fatty Liver Disease. J. Biochem. Mol. Toxicol. 2016, 30, 321-330. [CrossRef]

18. Ghose, R.; Omoluabi, O.; Gandhi, A.; Shah, P.; Strohacker, K.; Carpenter, K.C.; McFarlin, B.; Guo, T. Role of high-fat diet in regulation of gene expression of drug metabolizing enzymes and transporters. Life Sci. 2011, 89, 57-64. [CrossRef]

19. Quezada, C.; Alarcon, S.; Carcamo, J.G.; Yanez, A.; Casanello, P.; Sobrevia, L.; San Martin, R. Increased expression of the multidrug resistance-associated protein 1 (MRP1) in kidney glomeruli of streptozotocin-induced diabetic rats. Biol. Chem. 2011, 392, 529-537. [CrossRef] [PubMed]

20. Williams, M.J.; Wiemerslage, L.; Gohel, P.; Kheder, S.; Kothegala, L.V.; Schioth, H.B. Dibutyl Phthalate Exposure Disrupts Evolutionarily Conserved Insulin and Glucagon-Like Signaling in Drosophila Males. Endocrinology 2016, 157, $2309-2321$. [CrossRef] [PubMed]

21. Denholm, B.; Sudarsan, V.; Pasalodos-Sanchez, S.; Artero, R.; Lawrence, P.; Maddrell, S.; Baylies, M.; Skaer, H. Dual origin of the renal tubules in Drosophila: Mesodermal cells integrate and polarize to establish secretory function. Curr. Biol. 2003, 13, 1052-1057. [CrossRef]

22. Robinson, S.W.; Herzyk, P.; Dow, J.A.; Leader, D.P. FlyAtlas: Database of gene expression in the tissues of Drosophila melanogaster. Nucleic Acids Res. 2013, 41, D744-D750. [CrossRef]

23. Pfeiffenberger, C.; Lear, B.C.; Keegan, K.P.; Allada, R. Locomotor activity level monitoring using the Drosophila Activity Monitoring (DAM) System. Cold Spring Harb. Protoc. 2010, 2010, pdb.prot5518. [CrossRef] [PubMed]

24. Itskov, P.M.; Moreira, J.M.; Vinnik, E.; Lopes, G.; Safarik, S.; Dickinson, M.H.; Ribeiro, C. Automated monitoring and quantitative analysis of feeding behaviour in Drosophila. Nat. Commun. 2014, 5, 4560. [CrossRef] [PubMed]

25. Chintapalli, V.R.; Wang, J.; Dow, J.A. Using FlyAtlas to identify better Drosophila melanogaster models of human disease. Nat. Genet. 2007, 39, 715-720. [CrossRef]

26. Sieber, M.H.; Thummel, C.S. The DHR96 nuclear receptor controls triacylglycerol homeostasis in Drosophila. Cell Metab. 2009, 10, 481-490. [CrossRef]

27. Wang, D.Q. New concepts of mechanisms of intestinal cholesterol absorption. Ann. Hepatol. 2003, 2, 113-121. [CrossRef]

28. Whyte-Allman, S.K.; Hoque, M.T.; Jenabian, M.A.; Routy, J.P.; Bendayan, R. Xenobiotic Nuclear Receptors Pregnane X Receptor and Constitutive Androstane Receptor Regulate Antiretroviral Drug Efflux Transporters at the Blood-Testis Barrier. J. Pharmacol. Exp. Ther. 2017, 363, 324-335. [CrossRef] [PubMed]

29. Lucia, M.B.; Savarino, A.; Straface, E.; Golotta, C.; Rastrelli, E.; Matarrese, P.; Rutella, S.; Malorni, W.; Cauda, R. Role of lymphocyte multidrug resistance protein 1 in HIV infection: Expression, function, and consequences of inhibition. JAIDS J. Acquir. Immune Defic. Syndr. 2005, 40, 257-266. [CrossRef]

30. Bonilla, E.; Medina-Leendertz, S.; Villalobos, V.; Molero, L.; Bohorquez, A. Paraquat-induced oxidative stress in drosophila melanogaster: Effects of melatonin, glutathione, serotonin, minocycline, lipoic acid and ascorbic acid. Neurochem. Res. 2006, 31, 1425-1432. [CrossRef]

31. Huang, H.; Lu-Bo, Y.; Haddad, G.G. A Drosophila ABC transporter regulates lifespan. PLoS Genet. 2014, 10, e1004844. [CrossRef]

32. Kuzin, B.A.; Nikitina, E.A.; Cherezov, R.O.; Vorontsova, J.E.; Slezinger, M.S.; Zatsepina, O.G.; Simonova, O.B.; Enikolopov, G.N.; Savvateeva-Popova, E.V. Combination of hypomorphic mutations of the Drosophila homologues of aryl hydrocarbon receptor and nucleosome assembly protein family genes disrupts morphogenesis, memory and detoxification. PLoS ONE 2014, 9, e94975. [CrossRef]

33. Pitoniak, A.; Bohmann, D. Mechanisms and functions of Nrf2 signaling in Drosophila. Free Radic Biol. Med. 2015, 88, 302-313. [CrossRef] [PubMed]

34. Spiers, J.G.; Breda, C.; Robinson, S.; Giorgini, F.; Steinert, J.R. Drosophila Nrf2/Keap1 Mediated Redox Signaling Supports Synaptic Function and Longevity and Impacts on Circadian Activity. Front. Mol. Neurosci. 2019, 12, 86. [CrossRef] [PubMed]

35. Pohl, A.; Devaux, P.F.; Herrmann, A. Function of prokaryotic and eukaryotic ABC proteins in lipid transport. Biochim. Biophys. Acta Mol. Cell Biol. Lipids 2005, 1733, 29-52. [CrossRef] [PubMed]

36. Brand, A.H.; Perrimon, N. Targeted gene expression as a means of altering cell fates and generating dominant phenotypes. Development 1993, 118, 401-415. [CrossRef] [PubMed]

37. Soderberg, J.A.; Birse, R.T.; Nassel, D.R. Insulin production and signaling in renal tubules of Drosophila is under control of tachykinin-related peptide and regulates stress resistance. PLoS ONE 2011, 6, e19866. [CrossRef] 
38. Sun, J.; Usune, S.; Zhao, Y.; Migita, K.; Katsuragi, T. Multidrug resistance protein transporter and Ins(1,4,5) $\mathrm{P}(3)$-sensitive Ca(2)+signaling involved in adenosine triphosphate export via Gq protein-coupled NK(2)-receptor stimulation with neurokinin A. J. Pharmacol. Sci. 2010, 114, 92-98. [CrossRef] [PubMed]

39. Raggers, R.J.; van Helvoort, A.; Evers, R.; van Meer, G. The human multidrug resistance protein MRP1 translocates sphingolipid analogs across the plasma membrane. J. Cell Sci. 1999, 112 Pt 3, 415-422. [CrossRef]

40. Kamp, D.; Haest, C.W. Evidence for a role of the multidrug resistance protein (MRP) in the outward translocation of NBDphospholipids in the erythrocyte membrane. Biochim. Biophys. Acta Biomembr. 1998, 1372, 91-101. [CrossRef]

41. Cheng, Z.; Guo, S.; Copps, K.; Dong, X.; Kollipara, R.; Rodgers, J.T.; Depinho, R.A.; Puigserver, P.; White, M.F. Foxo1 integrates insulin signaling with mitochondrial function in the liver. Nat. Med. 2009, 15, 1307-1311. [CrossRef]

42. Sengupta, N.; Reardon, D.C.; Gerard, P.D.; Baldwin, W.S. Exchange of polar lipids from adults to neonates in Daphnia magna: Perturbations in sphingomyelin allocation by dietary lipids and environmental toxicants. PLoS ONE 2017, 12, e0178131. [CrossRef] [PubMed]

43. Elcombe, C.R.; Elcombe, B.M.; Foster, J.R.; Chang, S.C.; Ehresman, D.J.; Butenhoff, J.L. Hepatocellular hypertrophy and cell proliferation in Sprague-Dawley rats from dietary exposure to potassium perfluorooctanesulfonate results from increased expression of xenosensor nuclear receptors PPARalpha and CAR/PXR. Toxicology 2012, 293, 16-29. [CrossRef]

44. Dong, B.; Saha, P.K.; Huang, W.; Chen, W.; Abu-Elheiga, L.A.; Wakil, S.J.; Stevens, R.D.; Ilkayeva, O.; Newgard, C.B.; Chan, L.; et al. Activation of nuclear receptor CAR ameliorates diabetes and fatty liver disease. Proc. Natl. Acad. Sci. USA 2009, 106, 18831-18836. [CrossRef] [PubMed]

45. Kast, H.R.; Goodwin, B.; Tarr, P.T.; Jones, S.A.; Anisfeld, A.M.; Stoltz, C.M.; Tontonoz, P.; Kliewer, S.; Willson, T.M.; Edwards, P.A. Regulation of multidrug resistance-associated protein 2 (ABCC2) by the nuclear receptors pregnane $\mathrm{X}$ receptor, farnesoid X-activated receptor, and constitutive androstane receptor. J. Biol. Chem. 2002, 277, 2908-2915. [CrossRef]

46. Takahashi, K.; Shibata, T.; Oba, T.; Ishikawa, T.; Yoshikawa, M.; Tatsunami, R.; Takahashi, K.; Tampo, Y. Multidrug-resistanceassociated protein plays a protective role in menadione-induced oxidative stress in endothelial cells. Life Sci. 2009, 84, $211-217$. [CrossRef] [PubMed]

47. Takahashi, K.; Tatsunami, R.; Sato, K.; Tampo, Y. Multidrug resistance associated protein 1 together with glutathione plays a protective role against 4-hydroxy-2-nonenal-induced oxidative stress in bovine aortic endothelial cells. Biol. Pharm. Bull. 2012, 35, 1269-1274. [CrossRef]

48. Motohashi, H.; Yamamoto, M. Nrf2-Keap1 defines a physiologically important stress response mechanism. Trends Mol. Med. 2004, 10, 549-557. [CrossRef]

49. Sykiotis, G.P.; Bohmann, D. Keap1/Nrf2 signaling regulates oxidative stress tolerance and lifespan in Drosophila. Dev. Cell 2008, 14, 76-85. [CrossRef] [PubMed]

50. Zhang, X.; Moore, J.N.; Newsted, J.L.; Hecker, M.; Zwiernik, M.J.; Jones, P.D.; Bursian, S.J.; Giesy, J.P. Sequencing and characterization of mixed function monooxygenase genes CYP1A1 and CYP1A2 of Mink (Mustela vison) to facilitate study of dioxin-like compounds. Toxicol. Appl. Pharmacol. 2009, 234, 306-313. [CrossRef] [PubMed]

51. Aleksunes, L.M.; Klaassen, C.D. Coordinated regulation of hepatic phase I and II drug-metabolizing genes and transporters using AhR-, CAR-, PXR-, PPARalpha-, and Nrf2-null mice. Drug Metab. Dispos. 2012, 40, 1366-1379. [CrossRef] [PubMed]

52. Petrick, J.S.; Klaassen, C.D. Importance of hepatic induction of constitutive androstane receptor and other transcription factors that regulate xenobiotic metabolism and transport. Drug Metab. Dispos. 2007, 35, 1806-1815. [CrossRef] [PubMed]

53. Maher, J.M.; Cheng, X.; Slitt, A.L.; Dieter, M.Z.; Klaassen, C.D. Induction of the multidrug resistance-associated protein family of transporters by chemical activators of receptor-mediated pathways in mouse liver. Drug Metab. Dispos. 2005, 33, 956-962. [CrossRef] [PubMed] 